\title{
Paediatric medical outpatients: are all those reviews necessary?
}

\author{
K L Dodd, S Rhead, K Towey
}

\begin{abstract}
The value of and need for paediatric outpatient review attendance as perceived by parents, children, consultants, and general practitioners (GPs) were assessed.

One hundred and ninety one parents of 239 children over 7 years of age undergoing review were randomly selected for a semistructured interview. For each parent interviewed, an audit questionnaire was completed after case note review by another paediatrician. A random sample of the patients' GPs was surveyed by postal questionnaire.

Twenty per cent of parents and $26 \%$ of GPs felt that the GP could care for the child as well as or better than the hospital, whereas only $6 \%$ of consultants felt this to be so. Regarding future attendance of the child at the hospital, $48 \%$ of parents and $32 \%$ of GPs felt the child could either be discharged or seen when parents were worried, whereas consultants felt $24 \%$ of patients should have been discharged.

(Arch Dis Child 1994; 70: 493-496)
\end{abstract}

Outpatient work in paediatric medicine is becoming increasingly important as lengths of inpatient stays decrease and more emphasis is placed on preventing unnecessary admissions.

There is evidence of increasing workload in paediatric medical outpatient clinics, ${ }^{1}$ as well as in general medical clinics. ${ }^{2}$ Total attendances of paediatric outpatients in southern Derbyshire increased by $46 \%$ over the five year period 1984-9 from 7431 to 10835 . The ratio of review to new patients also increased from 3.6 to 4.95 in this period. This is still below the average ratio for the Trent region of $5 \cdot 25$ and that quoted by MacFaul and Long for Wakefield, $5 \cdot 75 .^{3}$

There is also evidence that the workload in paediatric outpatient clinics is high and patients are not offered sufficient time at an individual consultation. ${ }^{4}$ Other workers have found variations in the frequency with which patients with similar disorders are reviewed. ${ }^{3}$ This raises the question as to whether some children could be treated as effectively by their own family doctors.

A non-attendance rate of $35 \%$ was reported by Andrews et al for paediatric outpatient reviews at a district general hospital in Birmingham. ${ }^{5}$ Parents' perceptions of their child's symptoms, health, and prognosis influenced their decision not to attend.

The need for clinical audit is well established and it is important to take account of the views of both parents and children in assessing the service provided. The aims of this study were therefore to assess the value of, and need for, paediatric outpatient attendance as perceived by parents, children, consultants, and general practitioners (GPs) and then to suggest guidelines for continued attendance.

\section{Patients and methods \\ PATIENTS}

The study took place at Derbyshire Children's Hospital from June 1991 to December 1991. It covered the general paediatric medical outpatient clinics served by four consultant paediatricians and their registrars (including senior registrars, registrars, senior house officers, and clinical assistants), including a specialist renal clinic, but excluding clinics in diabetes and oncology. Patients attending for review were randomly selected at the time of the clinic, and the questionnaires administered immediately after their consultation with the doctor. The doctors were unaware which patients had been selected.

\section{METHODS}

The interviews were undertaken by one of the authors in a quiet room within the hospital away from the outpatient department.

Separate semistructured interviews were conducted for parents and for children over 7 years of age. The results of the interviews with children will be reported at a later date.

The medical notes were then forwarded to a second consultant paediatrician who had not seen the child for completion of an audit questionnaire using a standard protocol. In addition, a third of the total sample was randomly selected to assess the views of their GP by postal questionnaire.

\section{Results}

One hundred and ninety one of 239 parents of 239 children approached agreed to be interviewed, a response rate of $80 \%$. The case notes of all these children were audited by a consultant other than their own. The parents' reasons for not agreeing to participate included lack of time, other family commitments, and fractious children. Sixty one $(70 \%)$ of 87 GPs returned the questionnaires.

Table 1 gives the age distribution of the children attending and the division between consultant and registrar clinics. The children were fairly evenly distributed in the three age bands. A higher proportion of the registrar case load comprised children under 4 years of age. 
Table 1 Age distribution of children and their allocation to consultant and registrar clinics

\begin{tabular}{llcc}
\hline $\begin{array}{l}\text { Age } \\
\text { (years) }\end{array}$ & $\begin{array}{l}\text { No } \\
\text { (\%) }\end{array}$ & $\begin{array}{l}\text { Consultant } \\
\text { clinic (\%) }\end{array}$ & $\begin{array}{l}\text { Registrar } \\
\text { clinic (\%) }\end{array}$ \\
\hline $0-4$ & $75(39)$ & 35 & 49 \\
$5-8$ & $51(27)$ & 27 & 27 \\
$>8$ & $65(34)$ & 38 & 25 \\
Total & $191(100)$ & 100 & 100 \\
\hline
\end{tabular}

Table 2 gives the distribution of the sample by a simple grouping of diagnoses and the breakdown for consultant and registrar clinics. Children with epilepsy or renal problems were more likely to be seen by the consultant and those with asthma more likely to be seen by the registrar.

Parents, consultants, and GPs were asked for their opinions on the GP's ability to treat the child (table 3 ). Twenty per cent of parents and $26 \%$ of GPs felt the child could be treated just as well or better by the GP compared with only $6 \%$ of consultants.

This was followed by a question seeking views on the child's future attendance pattern (table 4). Forty eight per cent of parents and $32 \%$ of GPs would be happy for the child to be discharged or seen only if the parent was worried. Twenty four per cent of consultant audits felt the child could be discharged but in no case did a consultant feel the child should be seen only if parents were worried.

Numbers in the subgroups are small, but from both consultant audit and parents' views there was little difference between registrar and consultant clinics; however, GPs felt more could be discharged from the registrar than the consultant clinics. Those patients identified by consultant audit for discharge were twice as likely to have asthma as the study group as a whole and were also more likely to be attending a registrar clinic. The parents of children with asthma, renal, or miscellaneous disorders were more likely to opt to be 'seen when worried'.

Length of follow up and the number of doctors seen are also important variables. Although nearly a half ( $49 \%)$ of patients had been attending for over two years and a quarter $(24 \%)$ for over four years, most $(73 \%)$ had seen only one or two doctors. Children with a short length of follow up and especially those attending for less than two years were more likely to be considered suitable for discharge by the parents and consultant audit.

In the group of children whose parents felt they could be discharged but the consultant audit did not, however, those attending for more than four years were over represented, as

Table 2 Child attendances by diagnostic group and allocation to consultant and registrar clinics

\begin{tabular}{llll}
\hline $\begin{array}{l}\text { Diagnostic } \\
\text { group }\end{array}$ & $\begin{array}{l}\text { No } \\
(\%)\end{array}$ & $\begin{array}{l}\text { Consultant } \\
\text { clinic (\%) }\end{array}$ & $\begin{array}{l}\text { Registrar } \\
\text { clinic (\%) }\end{array}$ \\
\hline Asthma & $60(32)$ & 22 & 48 \\
Epilepsy & $38(20)$ & 11 & 3 \\
Bowels & $14(7)$ & 7 & 9 \\
Renal & $14(7)$ & 25 & 9 \\
Miscellaneous & $65(34)$ & 36 & 31 \\
\hline
\end{tabular}

^Includes abdominal pain, constipation, diarrhoea, and vomiting.
Table 3 Parents', consultants', and GPs' views on the GP's ability to treat the child. Values are number (\%)

\begin{tabular}{lccc}
\hline & Parents & Consultants & GPs \\
\hline Not as well & $145(76)$ & $180(94)$ & $45(74)^{\star}$ \\
Just as well & $35(18)$ & $11(6)$ & $13(21)$ \\
Better & $3(2)$ & $0(0)$ & $3(5)$ \\
Don't know & $8(4)$ & $0(0)$ & $0(0)$ \\
Total & $191(100)$ & $191(100)$ & $61(100)$ \\
\hline
\end{tabular}

^Fifty seven of GPs felt they could not have handled the problem as well alone but could have done so in cooperation with the hospital.

were those who had seen six or more doctors. Numbers in this subgroup are small but parental responses were more likely to indicate dissatisfaction with the visit, citing, for example, a perceived lack of interest of the doctor in their child.

\section{Discussion}

The study raises four main points. A major problem in mounting this study was the inadequacy of present hospital information systems related to outpatient activity. Routine data are not kept on age, diagnosis, or discharge rate. We were thus unable accurately to describe the population from which our study sample was taken, and it is therefore not possible to say if our study is a representative sample of patients attending general paediatric clinics. We did not investigate the $48(20 \%)$ of 239 parents who refused to participate in the study, nor those who did not attend the clinic, on average $27 \%$. Our findings therefore relate to a group of parents who took the trouble to attend the clinic and agreed to participate in the study. We suspect they were more likely to have been well motivated and favourably disposed towards future attendance than those who did not attend or refused to participate.

We were surprised that in only $6 \%$ of cases did the consultants think the GP could have handled the case as well as the hospital, compared with $20 \%$ of parents and $26 \%$ of GPs. Although it is possible that this question could have been misinterpreted in some cases, we suspect from subsequent discussion that this results in part from a paternalistic view of the paediatrician's role and implies a lack of confidence in the general practitioner.

The findings suggest that in the clinics studied, between one fifth and a quarter of review attendances were not necessary, based on the views of parents, GPs, and consultants. Their views did not always coincide. For example, of the patients considered by the consultants as suitable for discharge, a high

Table 4 Parents', consultants', and GPs'views on the child's future attendance. Values are number (\%)

\begin{tabular}{lccc}
\hline & Parents & Consultants & GPs \\
\hline Discharge & $28(15)$ & $46(24)$ & $13(22)$ \\
Stay as is & $83(43)$ & $134(70)$ & $28(47)$ \\
If worried & $64(34)$ & $0(0)$ & $6(10)$ \\
Less frequent & $8(4)$ & $7(4)$ & $3(5)$ \\
More frequent & $5(3)$ & $3(2)$ & $1(2)$ \\
Other & $3(2)$ & $1(1)$ & $9(15)^{\star}$ \\
Total & $191(100)$ & $191(100)$ & $60(100)$ \\
\hline
\end{tabular}

$\star$ Six GPs did not answer this question. 
proportion were children with asthma, but asthma was not over represented in the group of children whose parents felt they could be discharged. There appears to be a difference in perception of the need for follow up of children with asthma between parents and consultants, parents seeing asthma as a more threatening disorder demanding hospital follow up. Therefore if strategies are to be developed to discharge more children with asthma from outpatient follow up, clear protocols and guidelines agreed with GPs and better communication with parents would be needed. Monitoring follow up arrangements in registrar clinics has already been instituted.

In a study of a breast outpatient clinic in a district general hospital, Dash found up to $40 \%$ of appointments were potentially unnecessary in the view of the consultants and local GPs. ${ }^{6}$ Our study, which included the views of parents, found over $20 \%$ of appointments to be unnecessary. Correction of this should improve the service available to those who need to attend and help to meet standards of the patient's charter within existing resources. ${ }^{7}$

None of the four consultants considered seeing the child only if the parents were worried, an option favoured by one third of parents. It is not clear why this should be. Paediatricians commonly state that parents know their children best and that doctors should listen to and be guided by them. Many paediatricians are happy to encourage direct access for special groups of patients - for example, those with cystic fibrosis, cancer, and severe disabling disorders. Why are they reluctant to accept direct access in this study? A small proportion of GPs would have been happy with this arrangement and it therefore merits further discussion between paediatricians and GPs. Locally, our community paediatricians indicate they also wish to be involved. A potential problem is that direct access could lead to the development of a hospital based primary care system which might suit the parents and children, but could be open to abuse. Such arrangements should only be introduced with the agreement of the individual GP.

\section{Conclusions}

In spite of the fact that the clinics studied have a lower review rate than those in adjacent districts, there are still opportunities to reduce the workload in paediatric medical outpatient departments. This should be achieved by discussion between consultants and GPs and the development of agreed guidelines, especially for children with asthma and for those attending registrar clinics. The feasibility of direct access to outpatient clinics when parents are worried needs to be explored further.

1 Dawson KP, Ford RPK. Who comes to a paediatric medica outpatient clinic? NZ Med $\mathcal{F}$ 1988; 101: 111-2.

2 Samanta A, Haider Y, Roffe C. An audit of patients attending a general medical follow-up clinic. $f R$ Coll Physicians Lond 1991; 25: 33-5.

3 MacFaul R, Long R. Paediatric outpatient utilisation in district general hospital. Arch Dis Child 1992; 67: 1068-72.
4 Partridge JW. Consultation time, workload, and problems for audit in outpatient clinics. Arch Dis Child 1992; 67: 206-10.

5 Andrews $R$, Morgan JD, Addy DP, McNeish AS. Understanding non-attendance in outpatient paediatric clinics. Arch Dis Child 1990; 65: 192-5.

6 Dash P. Playing the waiting game. The Health Service fournal 1991; August: 16-7.

7 Collins C. Implementing the patient's charter in outpatient services. BMF 1992; 304: 1396.

\section{Commentary}

Paediatric outpatient services, which are almost exclusively hospital based, are overdue for searching examination. Many children are referred to outpatient clinics with disorders which are more appropriately dealt with in primary care or community paediatric clinics. ${ }^{1}$ Repeated reviews are not unusual, often by a different doctor on each occasion.

The paper of Dodd et al is a valuable contribution to the small number of publications on this important aspect of paediatric practice. They focus on parental, general practitioner, and consultant perceptions of the need for paediatric outpatient review attendance. The paper might be better entitled 'Paediatric medical outpatient reviews - who thinks they are necessary?'

\section{ROLE OF PARENTS}

The inclusion of parental perceptions is welcome. The findings of Dodd et al suggest that paediatricians remain reluctant to accept parents as skillful and responsible partners in the health care of their children, despite the strength of evidence ${ }^{23}$ and their apparent willingness to accept active parental involvement in, and direct parental access to, secondary and tertiary services for disorders such as cystic fibrosis. With the possible exception of asthma, parents, with the support of the general practitioners (GPs), wanted to be given more control over decision making related to the health of their children. A meaningful partnership with parents in the health care of their child requires that all professionals negotiate treatment and management decisions with parents and strive to develop services which reflect parental needs as much as those of service managers and professionals. ${ }^{4}$

\section{WHERE SHOULD PAEDIATRIC MEDICAL} OUTPATIENTS BE SEEN?

The role of GPs in the review of children referred to paediatric outpatient clinics raises the wider issue of where children referred for consultant paediatric opinion should be seen. A large percentage of the children seen in general paediatric medical outpatient clinics do not require investigation and many present with psychosocial, emotional and family problems, or normal variants. ${ }^{15}$ These children can be managed in community based clinics or GP surgeries to the advantage of the parent, child, and primary health care professionals. ${ }^{1}$ Joint consultations with GPs serve to strengthen primary care as well as allowing more rational and appropriate management to be negotiated between the parents, GPs, and 